\title{
FUNCTIONAL EQUATION OF A SPECIAL DIRICHLET SERIES
}

\author{
IBRAHIM A. ABOU-TAIR \\ Department of Mathematics \\ Islamic University - Gaza \\ Gaza - Strip \\ (Received December 10, 1985 and in revised form May 1, 1986)
}

ABSTRACT. In this paper we study the special Dirichlet series

$$
L(s)=\frac{2}{\sqrt{3}} \sum_{n=1}^{\infty} \sin \left(\frac{2 \pi n}{3}\right) n^{-s}, s \in C
$$

This series converges uniformly in the half-plane $\operatorname{Re}(s)>1$ and thus represents a holomorphic function there. We show that the function $L$ can be extended to a holomorphic function in the whole complex-plane. The values of the function $L$ at the points $0, \pm 1,-2, \pm 3,-4, \pm 5, \ldots$ are obtained. The values at the positive integers $1,3,5, \ldots$ are determined by means of a functional equation satisfied by $L$.

KEY WORDS AND PHRASES. Dirichlet Series, Analytic Continuation, Functional Equation, $\Gamma$-Function.

1980 AMS SUBJECT CLASSIFICATION CODE. 30B50, $30 B 40$.

1. INTRODUCTION.

By a Dirichlet series we mean a series of the form

$$
\sum_{n=1}^{\infty} a_{n^{n}} n^{-s}
$$

where the coefficients $a_{n}$ are any given numbers, and $s$ is a complex variable [1], [2].

In this paper we study the special Dirichlet series

$$
L(s)=\frac{2}{\sqrt{3}} \sum_{n=1}^{\infty} \sin \left(\frac{2 \pi n}{3}\right) n^{-s}, s \in C
$$

which converges uniformly in the half-plane $\operatorname{Re}(s)>1$ and thus represents an analytic function there. In section 1 we study the analytic behaviour of the function $L$ beyond the half-plane $\operatorname{Re}(s)>1$, and prove that the function $\mathrm{L}$ can be extended to a holomorphic function in the whole complex-plane. Moreover values of $L$ at the points $-m(m=0,1,2,3, \ldots)$ are obtained at the end of this section. The values of $L$ at the positive integers $1,3,5, \ldots$ are determined by means of the functional equation

$$
L(s)=\frac{2}{\sqrt{3}}\left(\frac{2 \pi}{3}\right)^{s-1} \Gamma(1-s) \cos \left(\frac{1}{2} \pi s\right) L(1-s), s<C
$$

satisfied by the function $\mathrm{L}$, which we prove in section 2 . 
2. ANALYTIC CONTINUATION OF L.

$$
L(s)=\frac{2}{\sqrt{3}} \sum_{n=1}^{\infty} \sin \left(\frac{2 \pi n}{3}\right) n^{-s}, \text { (s } \in C \text { ) }
$$

is uniformly convergent in the half-plane $\operatorname{Re}(s)>1$ and so it represents an analytic function there. The aim of this section is to extend $L$ to the whole complex plane and to prove that $L$ is holomorphic in $C$.

LEMMA 2.1. For all values of $s$ in the half-plane $\operatorname{Re}(s)>1$

$$
\begin{aligned}
L(s) & =\frac{1}{\Gamma(s)} \int_{0}^{\infty} G(t) t^{s-1} d t & & \text {, where } \\
G(t) & =\frac{2}{\sqrt{3}} \sum_{n=1}^{\infty} \sin \left(\frac{2 \pi n}{3}\right) e^{-n t} & & , \operatorname{Re}(t)>0 \\
& =\frac{1}{e^{t}+e^{-t}+1} & &
\end{aligned}
$$

PROOF. Cousider the Euler's integral.

$$
\Gamma(s)=\int_{0}^{\infty} e^{-t} t^{s-1} d t
$$

Substitution of $n t, n \in N$, for $t$ in the above integral yields

$$
n^{-s} \Gamma(s)=\int_{0}^{\infty} e^{-n t} t^{s-1} d t \quad, \operatorname{Re}(s)>0
$$

Thus for $\operatorname{Re}(s)>1$, we get

i.e.

$$
\Gamma(s) L(s)=\frac{2}{\sqrt{3}} \sum_{n=1}^{\infty} \sin \left(\frac{2 \pi n}{3}\right) \int_{0}^{\infty} e^{-n t} t^{s-1} d t
$$

Thus

$$
\Gamma(s) L(s)=\frac{2}{\sqrt{3}} \int_{0}^{\infty} \sum_{n=1}^{\infty} \sin \left(\frac{2 \pi n}{3}\right) e^{-n t} t^{s-1} d t
$$

Now

$$
\Gamma(s) L(s)=\int_{0}^{\infty} G(t) t^{s-1} d t
$$

i.e.

$$
G(t)=\frac{1}{i \sqrt{3}} \sum_{n=1}^{\infty}\left((\varepsilon)^{n}-(\bar{\varepsilon})^{n}\right) e^{-n t} \text {, where } \varepsilon=e^{2 \pi i / 3} \text {. }
$$

$$
G(t)=\frac{1}{i \sqrt{3}}\left(\sum_{n=1}^{\infty}(\varepsilon)^{n} e^{-n t}-\sum_{n=1}^{\infty}(\bar{\varepsilon})^{n} e^{-n t}\right), \operatorname{Re}(t)>0 .
$$

Thus

$$
G(t)=\frac{1}{i \sqrt{3}}\left(\frac{1}{\left(1-\varepsilon e^{-t}\right)}-\frac{1}{\left(1-\bar{\varepsilon} e^{-t}\right)}\right) \text {. }
$$

By using the identities $\varepsilon-\bar{\varepsilon}=i \sqrt{3}, \varepsilon+\bar{\varepsilon}+1=0$ and $\varepsilon \bar{\varepsilon}=1$, we get

$$
G(t)=\frac{1}{e^{t}+e^{-t}+1}
$$


The function $G(t)=\left(e^{t}+e^{-t}+1\right)^{-1}$ is analytic near $t=0$; therefore it can be expanded as a power series in $t$. So we have

LEMMA 2.2. $G(t)$ has the Taylor series expantion

$$
G(t)=\sum_{n=0}^{\infty} a_{n} t^{2 n} \quad,|t|<2 \pi / 3
$$

where the coefficients $a_{n}$ satisfy the recursion formula

$$
a_{0}=1 / 3,3 a_{n}+2 \sum_{k=1}^{n} \frac{1}{(2 k) !} a_{n-k}=0, n \geqslant 1
$$

PROOF. Since $G$ is an even function, the expantion of $G$ can be expressed as

$$
G(t)=\sum_{n=0}^{\infty} a_{n} t^{2 n}
$$

which is valid near zero (in fact valid in the disk $|t|<\frac{2}{3} \pi$ which extends to the nearest singularities $t= \pm \frac{2 \pi}{3}$ of $\left.G(t)\right)$. The relation $G(t)\left(e^{t}+e^{-t}+1\right)=1$ gives

$$
\left(\sum_{n=0}^{\infty} a_{n} t^{2 n}\right)\left(1+2 \sum_{n=0}^{\infty} \frac{t^{2 n}}{(2 n) !}\right)=1
$$

i.e.

$$
\sum_{n=0}^{\infty} a_{n} t^{2 n}+2\left(\sum_{n=0}^{\infty} a_{n} t^{2 n}\right)\left(\sum_{n=0}^{\infty} \frac{t^{2 n}}{(2 n) !}\right)=1
$$

i.e.

$$
\sum_{n=0}^{\infty} a_{n} t^{2 n}+2 \sum_{n=0}^{\infty}\left(\sum_{k=0}^{n} \frac{1}{(2 k) ! a_{n-k}}\right) t^{2 n}=1
$$

Thus for the coefficients $a_{n}$ we have the recursion formula

$$
a_{0}=1 / 3,3 a_{n}+2 \sum_{k=1}^{n} \frac{1}{(2 k) !} a_{n-k}=0, n \geqslant 1 .
$$

This completes the proof of the lemma.

The coefficient $a_{n}$ can be determined successively by (2.2). The first few are easily determined to be

$$
\begin{array}{ll}
a_{0}=\frac{1}{3}, & a_{1}=-\frac{1}{9} \\
a_{2}=\frac{1}{36}, & a_{3}=-\frac{7}{1080}
\end{array}
$$

THEOREM 2.1. The function $L$ defined by

$$
L(s)=\frac{1}{\Gamma(s)} \int_{0}^{\infty} G(t) t^{s-1} d t \quad, \operatorname{Re}(s)>1
$$

can be extended to a holomorphic function in the whole complex plane.

PROOF. Let us define $P$ and $Q$ for $\operatorname{Re}(s)>1$ by

$$
\begin{aligned}
& P(s)=\int_{0}^{1} G(t) t^{s-1} d t \\
& Q(s)=\int_{1}^{\infty} G(t) t^{s-1} d t
\end{aligned}
$$


The integral

$$
\int_{1}^{\infty} G(t) t^{s-1} d t
$$

exists and converges uniformly in any finite region of the s-plane, since the function

$$
\left(e^{-t} t^{\operatorname{Re}(s)+1}\right) /\left(e^{-t}+e^{-2 t}+1\right)
$$

is bounded for all values of $\operatorname{Re}(s)$, and we can compare the integral with that of $1 / t^{2}$. Thus $Q$ is an entire function. Recall from Lemma 2.2 that

$$
G(t)=\sum_{n=0}^{\infty} a_{n} t^{2 n} \quad, t \in[0,1]
$$

the convergence being uniform on $[0,1]$. We deduce for $\operatorname{Re}(s)>1$ that

$$
\begin{aligned}
P(s) & =\sum_{n=0}^{\infty} \int_{0}^{1} a_{n} t^{2 n+s-1} d t \\
& =\sum_{n=0}^{\infty} \frac{1}{2 n+s} a_{n}
\end{aligned}
$$

Thus $P$ is a meromorphic function on $C$ with simple poles at $0,-2,-4,-6, \ldots$. Since $1 / \Gamma$ is an entire function we may now extend $L$ to the whole of $C$ by

$$
L(s)=\frac{P(s)}{\Gamma(s)}+\frac{Q(s)}{\Gamma(s)}
$$

Since $Q$ and $1 / \Gamma$ are entire functions, the singularities of $L$ can only be those of $P / \Gamma$. We have seen that $P$ has simple poles at $0,-2,-4,-6, \ldots$. Since $1 / \Gamma$ has simple zeros at $0,-2,-4, \ldots$ it follows that $L$ is regular for all values of $s$ in the complex plane. This completes the proof of the theorem.

LEMMA 2.3. (i) $L$ has zeros at $-1,-3,-5, \ldots$

(ii) The values of $\mathrm{L}$ at $0,-2,-4,-6, \ldots$ are given by

$$
L(-2 m)=(2 m) ! a_{m} \quad, m=0,1,2,3,4, \ldots
$$

PROOF. (i) This follows immediately from the fact that $1 / \Gamma$ has zeros at $0,-1$, $-2,-3, \ldots$, and thus

$$
L(1-2 m)=\frac{P(1-2 m)}{\Gamma(1-2 m)}+\frac{Q(1-2 m)}{\Gamma(1-2 m)}=0, m \in N .
$$

(ii) As in (i) we use the partial fraction (2.3) of $L$ to get

$$
\begin{aligned}
L(-2 m) & =\lim _{s \rightarrow-2 m} \frac{P(s)}{\Gamma(s)}+\frac{Q(s)}{\Gamma(s)} \\
& =\lim _{s \rightarrow-2 m} \frac{P(s)}{\Gamma(s)}=\lim _{s \rightarrow-2 m} \frac{1}{\Gamma(s)} \sum_{n=0}^{\infty} \frac{1}{2 n+s} a_{n}
\end{aligned}
$$

i.e.

$$
L(-2 m)=\lim _{s \rightarrow-2 m} \frac{1}{\Gamma(s)} \cdot \frac{1}{2 m+s} a_{m}
$$


Since $\Gamma$ has simple poles at the points $-m(m=0,1,2,3, \ldots)$ with residues $(-1)^{m} / m !$, we get

$$
\lim _{s \rightarrow-2 m}(2 m+s) \Gamma(s)=\operatorname{Res}(\Gamma,-2 m)=\frac{1}{(2 m) !}
$$

Thus

$$
L(-2 m)=(2 m) ! a_{m} \quad, m=0,1,2,3, \ldots
$$

where $a_{m}$ can be determined successively by (2.2).

3. DERIVATION OF THE FUNCTIONAL EQUATION OF L.

In this section we derive the equation

$$
L(s)=\frac{2}{\sqrt{3}}\left(\frac{2}{3} \pi\right)^{s-1} \Gamma(1-s) \cos \left(\frac{1}{2} \pi s\right) L(1-s), s \in C .
$$

where $\mathcal{L}$ is the Dirichlet series (2.1)

$$
L(s)=\frac{2}{\sqrt{3}} \sum_{n=1}^{\infty} \sin \left(\frac{2 \pi n}{3}\right) n^{-s}, \quad s \in C
$$

Finally we determine the values of $L$ at $1,3,5, \ldots$, by the use of the functional equation obtained above.

LEMMA 3.1. There exists an integral function I such that

$$
L(s)=-\Gamma(1-s) I(s) \quad, s \in C \text {. }
$$

PROOF. Let $0<r<1$, and let $C_{r}$ be the contour consisting of the paths $C_{1}$, $\mathrm{C}_{2}$ and $\mathrm{C}_{3}$, where

$C_{1}=(\infty, r]$

$C_{2}=\partial_{+} D_{r}(0)$ is a circle of radius $r$ and the center at the origin oriented in the positive direction.

$c_{3}=[r, \infty)$.

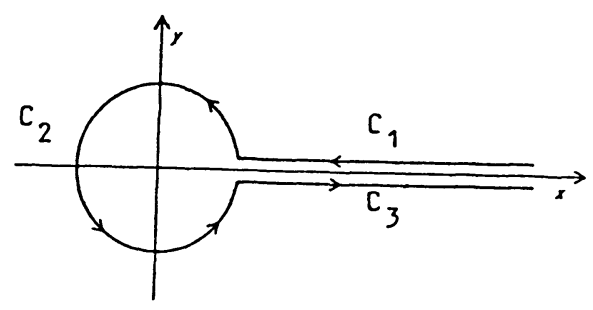

Define the function $I_{r}$ by

$$
I_{r}(s)=\frac{1}{2 \pi i} \int_{C_{r}} \frac{(-t)^{s-1}}{e^{t}+e^{-t}+1} d t
$$

We prove now that $I_{r}$ is independent of $r$. We have

$$
I_{r}(s)-I_{r} \cdot(s)=\frac{1}{2 \pi i} \int_{C_{0}} \frac{(-t)^{s-1}}{e^{t}+e^{-t}+1},
$$


where $C_{0}$ is the contour shown in figure (a). Now

$$
\int_{C_{0}} \frac{(-t)^{s-1}}{e^{t}+e^{-t}+1} d t=\lim _{\delta \rightarrow 0} \int_{C} \frac{(-t)^{s-1}}{e^{t}+e^{-t}+1} d t,
$$

where $C$ is the contour in figure (b).

According to Cauchy's theorem, the integral around $C$ is zero. Thus

$$
\int_{0} \frac{(-t)^{s-1}}{e^{t}+e^{-t}+1} d t=0
$$

It follows that $I_{r}$ is independent of $r$.

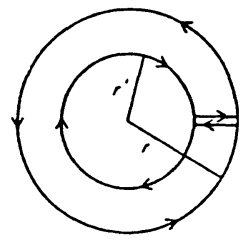

(a)

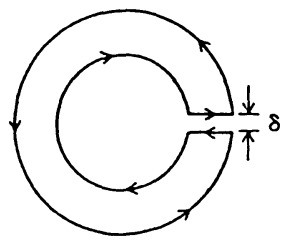

(b)

Now,

$$
\begin{gathered}
I_{r}(s)=\frac{1}{2 \pi i} \int_{\infty}^{r} \frac{e^{(\log t-\pi i)(s-1)}}{e^{t}+e^{-t}+1} d t+ \\
\frac{1}{2 \pi i} \int_{c_{2}} \frac{(-t)^{s-1}}{e^{t}+e^{-t}+1} d t+\frac{1}{2 \pi i} \int_{r}^{\infty} \frac{e^{(\log t+\pi i)(s-1)}}{e^{t}+e^{-t}+1} .
\end{gathered}
$$

The middle term approaches zero as $r \rightarrow 0$ provided $\operatorname{Re}(\mathrm{s})>0$, since

$$
\begin{aligned}
\left|\int_{C_{2}} \frac{(-t)^{s-1}}{e^{t}+e^{-t}+1} d t\right| & <M \int_{0}^{2 \pi} r^{R e(s)-1} e^{-(\pi+\theta) I m(s)} r d \theta \\
& <M^{\prime} r^{R e(s)} .
\end{aligned}
$$

Hence

$$
\lim _{r \rightarrow 0} I_{r}(s): \frac{-e^{-\pi i(s-1)}+e^{\pi i(s-1)}}{2 \pi i} \int_{0}^{\infty} \frac{t^{s-1}}{e^{t}+e^{-t}+1} d t .
$$

Define the function I by

$$
I(s)=\lim _{r \rightarrow 0} I_{r}(s)
$$

Thus we have

$$
I(s)=-\frac{\sin (\pi s)}{\pi} \int_{0}^{\infty} \frac{t^{s-1}}{e^{t}+e^{-t}+1} d t .
$$

We have seen in the proof of theorem 2.1 that the function defined by the integral

$$
\int_{0}^{\infty} \frac{t^{s-1}}{e^{t}+e^{-t}+1}
$$

is a meromorphic function with simple poles at the points $0,-2,-4, \ldots$. Since the function $\sin (\pi s)$ has simple zeros at $0,-2,-4, \ldots$ it follows that $I$ is regular for 
all values of $s$ in the complex plane.

Moreover we have

$$
I(s)=-\frac{\Gamma(s) \sin (\pi s)}{\pi} L(s)
$$

Thus

$$
I(s) \Gamma(1-s)=-L(s)
$$

THEOREM 3.1. The function $L$ satisfies the functional equation

$$
L(s)=\frac{2}{\sqrt{3}}\left(\frac{2}{3} \pi\right)^{s-1} \Gamma(1-s) \cos \left(\frac{1}{2} \pi s\right) L(1-s)
$$

PROOF. Let $R_{n}=n+\frac{1}{2}, n=1,2,3, \ldots$, and let $c_{n, r}(0<r<1)$ be the contour consisting of the positive real axis from $R_{n}$ to $r$, a circle radius $r$ and center at the origin oriented in the positive direction, the positive real axis from $r$ to $R_{n}$, and finally a circle of radius $R_{n}$ with center at the origin oriented in the negative direction.

1.e.

$$
c_{n, r}=\left[R_{n}, r\right]+\underset{+}{\partial} D_{r}(0)+\left[r, R_{n}\right]+\underset{\partial}{\partial} D_{R_{n}}(0) .
$$

To deduce the functional equation of $L$ we evaluate the integral

$$
\frac{1}{2 \pi i} \int_{C_{r, n}} \frac{(-t)^{s-1}}{e^{t}+e^{-t}+1} d t
$$

If we assume $s=x$ is a negative real number, then we have

$$
(-t)^{x-1}=e^{(x-1) \log (-t)}
$$

It follows that

$$
|(-t)|^{x-1}=|t|^{x-1}
$$

Since the function $\left(e^{t}+e^{-t}+1\right)^{-1}$ is bounded on the circle $\underline{\partial}_{R_{n}}(0)$,

$$
\left|\int_{-D_{R_{n}}(0)} \frac{(-t)^{s-1}}{e^{t}+e^{-t}+1}\right|<2 M^{*} R_{n}^{x} \text {, }
$$

which goes to zero as $\mathrm{n}$ goes to infinity.

Thus we have

$$
I(s)=\lim _{n \rightarrow \infty}\left(\frac{1}{2 \pi i} \int_{C_{n, r}} \frac{(-t)^{s-1}}{e^{t}+e^{-t}+1} d t\right) .
$$

Now between $\partial \mathrm{D}_{\mathrm{R}_{\mathrm{n}}}(0)$ and $\mathrm{D}_{\mathrm{r}}(0)$ the integrand has poles at the points

$$
\pm \frac{2 \pi i}{3}, \pm \frac{2 \pi i}{3}(3 m+1) \text { and } \pm \frac{2 \pi i}{3}(3 m-1), m=1,2,3, \ldots
$$


Denote

Thus we have

$$
H(t)=\frac{(-t)^{s-1}}{e^{t}+e^{-t}+1}
$$

$$
\begin{aligned}
& \operatorname{Res}\left(H, \frac{2 \pi i}{3}\right)=\frac{1}{\sqrt{3}}\left(\frac{2 \pi}{3}\right)^{s-1} \mathrm{e}^{-\pi i s / 2} . \\
& \operatorname{Res}\left(H,-\frac{2 \pi i}{3}\right)=\frac{1}{\sqrt{3}}\left(\frac{2}{3} \pi\right)^{s-1} \mathrm{e}^{\pi i s / 2} . \\
& \operatorname{Res}\left(H, \frac{2 \pi i}{3}(3 m+1)\right)=\frac{1}{\sqrt{3}}\left(\frac{2}{3} \pi\right)^{s-1} \mathrm{e}^{-\pi i s / 2}(3 m+1)^{s-1} . \\
& \operatorname{Res}\left(H,-\frac{2 \pi i}{3}(3 m+1)\right)=\frac{1}{\sqrt{3}}\left(\frac{2}{3} \pi\right)^{s-1} \mathrm{e}^{\pi i s / 2}(3 m+1)^{s-1} . \\
& \operatorname{Res}\left(H, \frac{2 \pi i}{3}(3 m-1)\right)=-\frac{1}{\sqrt{3}}\left(\frac{2}{3} \pi\right)^{s-1} \mathrm{e}^{-\pi i s / 2}(3 m-1)^{s-1} . \\
& \operatorname{Res}\left(H,-\frac{2 \pi i}{3}(3 m-1)=-\frac{1}{\sqrt{3}}\left(\frac{2}{3} \pi\right)^{s-1} \mathrm{e}^{\pi i s / 2}(3 m-1)^{s-1} .\right.
\end{aligned}
$$

The sum of the residues between $\partial D_{R_{n}}(0)$ and $\partial D_{r}(0)$ equals

One can easily verify the identity

$$
\frac{2}{\sqrt{3}}\left(\frac{2}{3} \pi\right)^{s-1} \cos \left(\frac{1}{2} \pi s\right)\left(1+\sum_{m=1}^{n}\left[(3 m+1)^{s-1}-(3 m-1)^{s-1}\right]\right)
$$

$$
1+\sum_{m=1}^{n}\left[(3 m+1)^{s-1}-(3 m-1)^{s-1}\right]=\frac{2}{\sqrt{3}} \sum_{m=1}^{3 n+1} \sin \left(\frac{2}{3} \pi m\right) m^{s-1} .
$$

Thus the sum of the residues is

$$
\frac{2}{\sqrt{3}}\left(\frac{2}{3} \pi\right)^{s-1} \cos \left(\frac{1}{2} \pi s\right)\left(\frac{2}{\sqrt{3}} \sum_{m=1}^{3 n+1} \sin \left(\frac{2}{3} \pi m\right) m^{s-1}\right)
$$

It follows that

$$
\begin{aligned}
-I(s) & =\frac{2}{\sqrt{3}}\left(\frac{2}{3} \pi\right)^{s-1} \cos \left(\frac{1}{2} \pi s\right)\left(\frac{2}{\sqrt{3}} \sum_{m=1}^{\infty} \sin \left(\frac{2}{3} \pi m\right) m^{s-1}\right) . \\
& =\frac{2}{\sqrt{3}}\left(\frac{2}{3} \pi\right)^{s-1} \cos \left(\frac{1}{2} \pi s\right) L(1-s)
\end{aligned}
$$

We have seen that $-I(s) \Gamma(1-s)=L(s)$ for all $s \in C$, so by the identity theorem the formula (3.1) is true for all $s \in C$. Thus we have proved the functional equation

$$
L(s)=\frac{2}{\sqrt{3}}\left(\frac{2}{3} \pi\right)^{s-1} \cos \left(\frac{1}{2} \pi s\right) \Gamma(1-s) L(1-s) .
$$

LEMMA 3.2. The values of $L$ at the points $s=2 m+1, m=0,1,2,3, \ldots$ are given by the formula

$$
L(1+2 m)=(-1)^{m} \frac{\sqrt{3}}{2}\left(\frac{2}{3} \pi\right)^{2 m+1} a_{m} .
$$

where $a_{m}$ 's are determined by (2.2). 
PROOF. For $s=-2 m$ the functional equation and the identity

$$
L(-2 m)=(2 m) ! a_{m}, m=0,1,2, \ldots
$$

of the previous section give the proof of the lemma.

\section{REFERENCES}

1. HARDY, G.H. and RIESZ, M. "The Genera1 Theory of Dirichlet Series," Cambridge University Press, 1952.

2. TITCHMARSH, E.C. "The Theory of the Riemann Zeta-Function," Oxford University Press, 1951. 


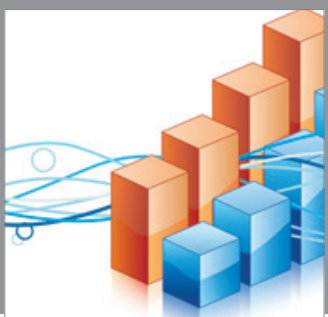

Advances in

Operations Research

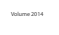

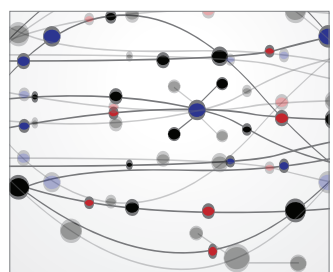

\section{The Scientific} World Journal
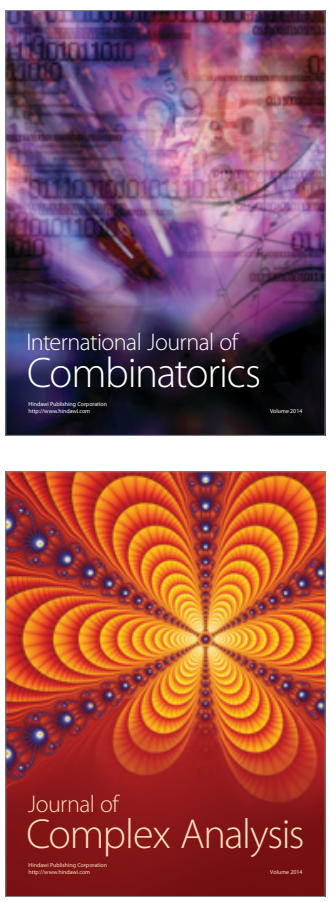

International Journal of

Mathematics and

Mathematical

Sciences
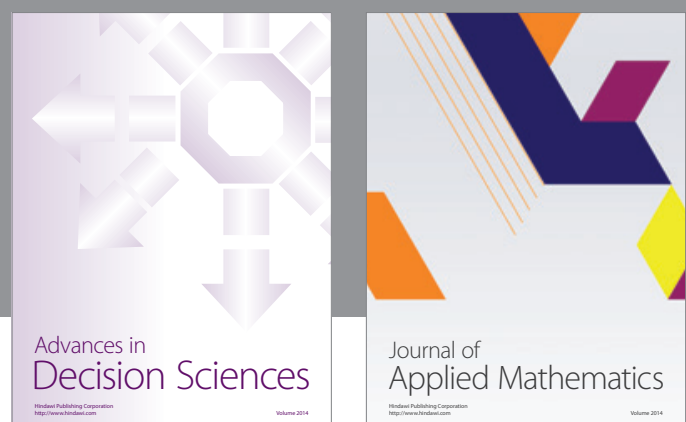

Journal of

Applied Mathematics
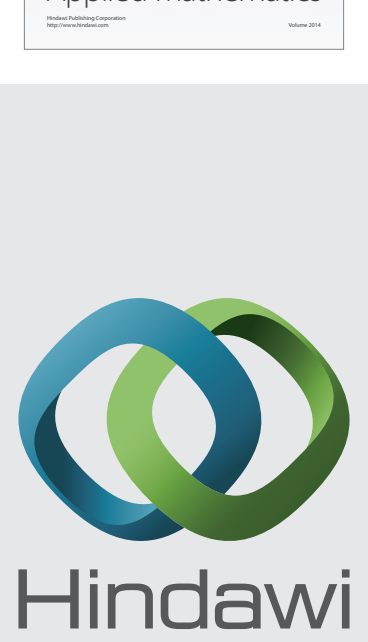

Submit your manuscripts at http://www.hindawi.com
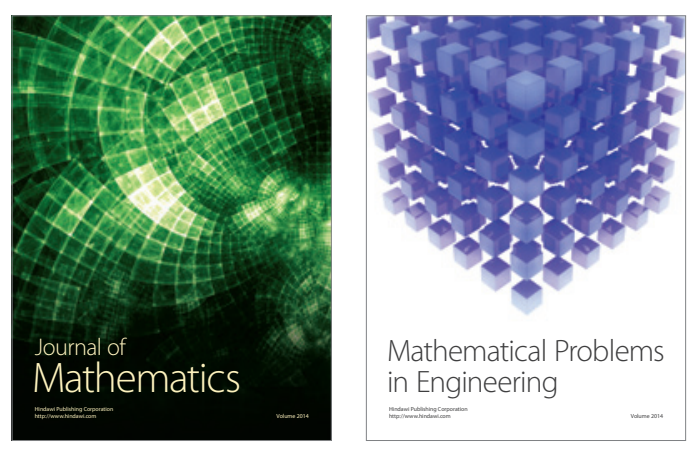

Mathematical Problems in Engineering
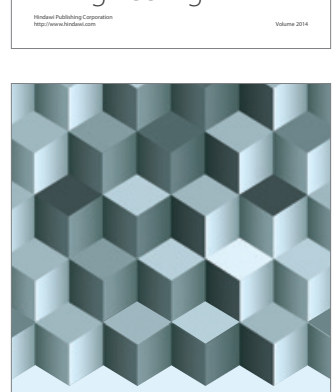

Journal of

Function Spaces
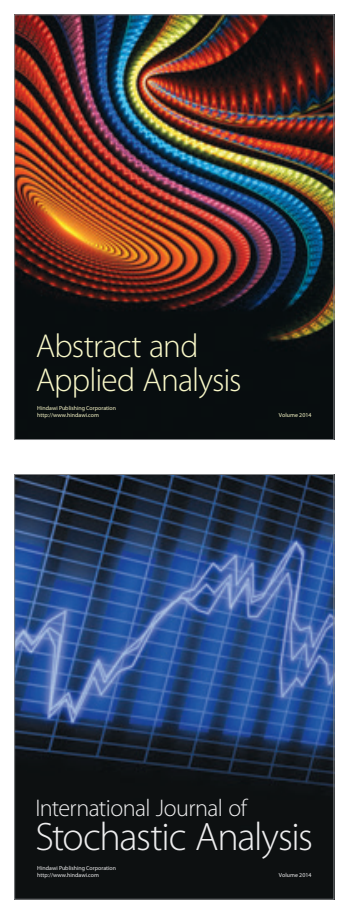

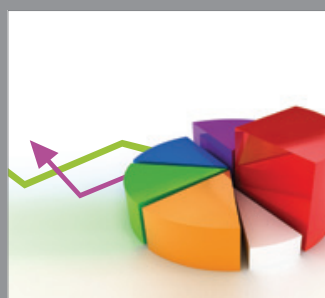

ournal of

Probability and Statistics

Promensencen
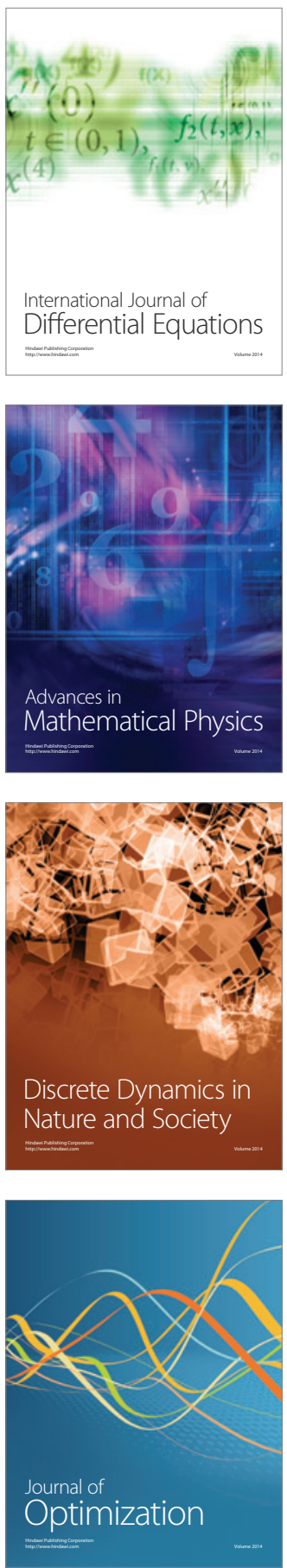